\title{
Light Hyperon Physics at BESIII
}

\author{
Viktor Thorén (on behalf of the BESIII collaboration) ${ }^{a, *}$ \\ ${ }^{a}$ Uppsala University, \\ Box 516, SE-75120, Uppsala, Sweden
}

E-mail: viktor.thoren@physics.uu.se

With the large datasets on $e^{+} e^{-}$-annihilation at the $J / \psi$ and $\psi^{\prime}$ resonances collected at the BESIII experiment, multi-dimensional analyses making use of polarization and entanglement can shed new light on the production and decay properties hyperon-antihyperon pairs. In a series of recent studies performed at BESIII, significant transverse polarization of the (anti)hyperons has been observed in $J / \psi$ or $\psi^{\prime}$ to $\Lambda \bar{\Lambda}, \Sigma^{+} \bar{\Sigma}^{-}, \Xi^{-} \bar{\Xi}^{+}$, and $\Omega^{-} \bar{\Omega}^{+}$and the spin of $\Omega^{-}$has been determined model-independently for the first time. The decay parameters for the most common hadronic weak decay modes were measured, and due to the non-zero polarization, the parameters of hyperon and antihyperon decays could be determined independently of each other for the first time. Comparing the hyperon and antihyperon decay parameters yields precise tests of direct, $\Delta S=1 \mathrm{CP}$-violation that complement studies performed in the kaon sector.

*** The European Physical Society Conference on High Energy Physics (EPS-HEP2021), ***

*** 26-30 July $2021 * * *$

*** Online conference, jointly organized by Universität Hamburg and the research center DESY ***

${ }^{*}$ Speaker 


\section{Introduction}

The well defined initial state of hyperon-antihyperon pairs produced at $e^{+} e^{-}$-colliders are excellent laboratories for tests of fundamental discrete symmetries such as CP. A formalism that describes the production via a single intermediate virtual photon and subsequent decays has been known for some time [1-5], and it has recently been shown that the same formalism also holds for intermediate vector resonances such as $J / \psi$ and $\psi^{\prime}$ [6]. In order to extract the decay properties, one must study polarizations and spin correlations through analysis of a multidimensional angular distributions. Due to low cross sections, such studies are not possible in continuum production, but thanks to the comparatively large branching fractions of $J / \psi \rightarrow B \bar{B}$, such studies are feasible using the datasets of $10^{10} \mathrm{~J} / \psi$ and $3 \times 10^{9} \psi^{\prime}$ events collected by the BESIII experiment.

\section{Hyperon Decays and Direct CP-violation}

Direct, $\Delta S=1 \mathrm{CP}$-violation has been observed in decays of kaons into two pions [7-9]. The CP-violating effect is observable because of the presence of two interfering amplitudes with different CP-odd phases corresponding to isopspin transitions $\mathcal{A}_{\Delta I=1 / 2}$ and $\mathcal{A}_{\Delta I=3 / 2}$. The size of the CP-violating contribution is quantified by the ratio of the partial decay widths of $K_{L}$ and $K_{S}$

$$
\frac{\mathcal{A}\left(K_{L} \rightarrow \pi^{0} \pi^{0}\right)}{\mathcal{A}\left(K_{S} \rightarrow \pi^{0} \pi^{0}\right)}:=\epsilon-2 \epsilon^{\prime} \text { and } \frac{\mathcal{A}\left(K_{L} \rightarrow \pi^{+} \pi^{-}\right)}{\mathcal{A}\left(K_{S} \rightarrow \pi^{+} \pi^{-}\right)}:=\epsilon+\epsilon^{\prime} .
$$

The observed values for the parameters $\epsilon^{\prime}$ and $\epsilon$ are consistent with the standard model (SM) where the effect originates from the CKM mixing matrix via the so-called penguin diagrams, see e.g. Ref. [10].

All ground state hyperons decay mainly via $\Delta S=1$ weak decays into a baryon and a meson. Since hyperons carry spin, there are parity-even $p$-wave and parity-odd $s$-wave contributions with amplitudes $P$ and $S$, respectively, for both isospin $\Delta I=1 / 2$ and $\Delta I=3 / 2$ transitions [11]. The full decay amplitude is given by

$$
\mathcal{A}=S+P \sigma \cdot \hat{n}
$$

where $\hat{n}$ is the direction of the daughter baryon momentum in the parent momentum rest frame. The $\Delta I=3 / 2$ transition is suppressed and to first order it is sufficient to consider the $\Delta I=1 / 2$ transition. This allows us to express the amplitude in terms of weak CP-odd phases $\xi$, and strong phases $\delta$, as

$$
\begin{aligned}
& S=|S| \exp \left(i \xi_{S}\right) \exp \left(i \delta_{S}\right) \\
& P=|P| \exp \left(i \xi_{P}\right) \exp \left(i \delta_{P}\right) .
\end{aligned}
$$

A non-zero weak phase difference $\left|\xi_{S}-\xi_{P}\right|$ would lead to CP-violation. The decay process can be described by two parameters $\alpha$ and $\beta$ defined in terms of the interference of the two amplitudes [12]

$$
\begin{aligned}
& \alpha=\frac{2 \operatorname{Re}\left(S^{*} P\right)}{|S|^{2}+|P|^{2}} \\
& \beta=\frac{2 \operatorname{Im}\left(S^{*} P\right)}{|S|^{2}+|P|^{2}}=\sqrt{1-\alpha^{2}} \sin \phi,
\end{aligned}
$$


where $\phi$ is a more natural choice for the experimentalist than $\beta$. The $\alpha$ parameter can be extracted in any one-step decay if both the polarization of the decaying hyperon and the angular distribution of its daughter baryon can be measured. Measuring $\phi$ on the other hand requires a two-step process such as $\Xi^{-} \rightarrow \Lambda \pi^{-}, \Lambda \rightarrow p \pi^{-}$where the polarization of sequentially decaying mother and daughter hyperons can be compared.

If $\mathrm{CP}$ is conserved, the decay parameters for baryon and antibaryon should have the same absolute value, but with opposite sign $\bar{\alpha}=-\alpha, \bar{\beta}=-\beta, \bar{\phi}=-\phi$. We can define two independent CP-tests

$$
\begin{aligned}
& A_{C P}=\frac{\alpha+\bar{\alpha}}{\alpha-\bar{\alpha}} \\
& B_{C P}=\frac{\beta+\bar{\beta}}{\alpha-\bar{\alpha}}=\left(\xi_{P}-\xi_{S}\right)
\end{aligned}
$$

The largest CP-violating contributions are expected in the decays $\Xi^{-} \rightarrow \Lambda \pi^{-}$and $\Lambda \rightarrow p \pi^{-}$, where the SM predictions for $A_{C P}$ are on the order of $10^{-5}$ [13]. Measurements of these observables can be used to constrain beyond SM contributions to $\epsilon$ and $\epsilon^{\prime}$ of the kaon sector and vice versa [14].

The HyperCP experiment has measured the combination $A_{C P}^{\Xi}+A_{C P}^{\Lambda}=0(5)(5) \times 10^{-4}[15]$, and the PS185 experiment has determined $A_{C P}^{\Lambda}=0.013 \pm 0.021$ by measuring the product of $\alpha_{\Lambda}$ and the polarization [16]. Until now, no independent measurements of the decay parameters of hyperons and antihyperons have been performed.

\section{Hyperon-Antihyperon Pair Production in $e^{+} e^{-}$Collisions}

Baryons produced in $e^{+} e^{-}$-annihilation can be polarized in the direction perpendicular to the production plane even if the beams are unpolarized. The degree of polarization, if any, depends on the production mechanism. The production of a spin-1/2 baryon-antibaryon pair $e^{+} e^{-} \rightarrow B \bar{B}$, via single-photon exchange or an intermediate vector meson $\left(\right.$ e.g. $\left.J / \psi, \psi^{\prime}\right)$, can be described by two complex form factors $G_{E}^{\psi}$ and $G_{M}^{\psi}$. [6]. These are quantified in terms of two observable parameters: $\alpha_{\psi}$, representing the ratio between the form factors and $\Delta \Phi$, the relative phase between them. These parameters are related to the scattering angle and polarization of the baryons. If $\Delta \Phi$ is non-zero, the baryons acquire a non-zero transverse polarization. Only if this is the case can the decay parameters of hyperon and antihyperon be determined independently from each other. The production and decay of a pair of spin-3/2 baryons is described by four complex form factors.

The production and subsequent decay of spin-1/2 or spin-3/2 baryon-antibaryon pairs and any combinations thereof can be described by a modular approach developed in Ref. [17]. The spin correlations and polarization are given by the spin density matrix given, for spin-1/2, by

$$
\rho_{B \bar{B}}=\sum C_{\mu \nu}^{1 / 2} \sigma_{\mu}^{B} \otimes \sigma_{v}^{\bar{B}}
$$

where $\sigma_{\mu}^{B}\left(\sigma_{v}^{\bar{B}}\right)$ are the Pauli matrices in the (anti)baryon helicity frame. The coefficient matrix $C_{\mu \nu}$ contains the information on the spin correlations $C_{i j}(\theta)$ and polarization $P_{y}(\theta)$. For a spin- $1 / 2$ 
baryon-antibaryon pair, it takes the form

$$
\begin{aligned}
C_{\mu \nu}^{1 / 2} & =3 \frac{1+\alpha_{\psi} \cos ^{2} \theta}{3+\alpha_{\psi}}\left(\begin{array}{cccc}
1 & 0 & P_{y} & 0 \\
0 & C_{x x} & 0 & C_{x z} \\
-P_{y} & 0 & C_{y y} & 0 \\
0 & -C_{x z} & 0 & C_{z z}
\end{array}\right) \\
& =\frac{3}{3+\alpha_{\psi}}\left(\begin{array}{cccc}
1+\alpha_{\psi} \cos ^{2} \theta & 0 & \beta_{\psi} \sin \theta \cos \theta & 0 \\
0 & \sin ^{2} \theta & 0 & \gamma_{\psi} \sin \theta \cos \theta \\
-\beta_{\psi} \sin \theta \cos \theta & 0 & \alpha_{\psi} \sin ^{2} \theta & 0 \\
0 & -\gamma_{\psi} \sin \theta \cos \theta & 0 & -\alpha_{\psi}-\cos ^{2} \theta
\end{array}\right),
\end{aligned}
$$

where $\beta_{\psi}=\sqrt{1-\alpha_{\psi}^{2}} \sin (\Delta \Phi)$ and $\gamma_{\psi}=\sqrt{1-\alpha_{\psi}^{2}} \cos (\Delta \Phi)$. The transformation of the spin operators in the hadronic two-body decays $D(B \rightarrow b \pi)$ are handled by decay matrices that depend on the parameters $\alpha$ and $\phi$

$$
\sigma_{\mu}^{B} \rightarrow \sum_{\nu=0}^{3} a_{\mu, v}^{D} \sigma_{v}^{b}
$$

Together, these allow for a very compact expression for the full angular distribution for any baryonantibaryon pair, including sequential decays. The full angular distribution for a spin-1/2 baryonantibaryon pair decaying in one step is given by

$$
\mathcal{W}(\xi, \omega)=\sum_{\mu, \nu=0}^{3} C_{\mu \nu} a_{\mu 0}^{D} a_{\nu 0}^{\bar{D}} .
$$

where $\xi$ is the set of helicity angles that completely describes an event and $\omega:=\left(\alpha_{\psi}, \Delta \Phi, \alpha, \bar{\alpha}\right)$ is the set of production and decay parameters. Sequential decays are taken into account by the application of additional decay matrices that each introduces a number of additional parameters and angular dependences.

\section{Experimental Method}

The BESIII experiment has collected the world's largest datasets at the $J / \psi$ and $\psi^{\prime}$ resonances with a total of $10^{10}$ and $3 \times 10^{9}$ events, respectively. The results presented in this contribution are based on $1.31 \times 10^{9} \mathrm{~J} / \psi$ and $448 \times 10^{6} \psi^{\prime}$ events. The process $J / \psi, \psi^{\prime} \rightarrow B \bar{B}$ can be reconstructed in two ways: double-tag (DT) where both baryon and antibaryon are reconstructed, and single-tag (ST) where either the baryon or the antibaryon is reconstructed and the missing mass is used to ensure that the events originate from the correct reaction.

For each signal event, all relevant helicity angles are determined, whereafter an unbinned maxmimum-log-likelihood (MLL) fit is used to determine the production and decay parameters. The detection efficiency as a function of all helicity angles is taken into account in the fit. 


\section{Recent Results from BESIII}

$5.1 e^{+} e^{-} \rightarrow J / \psi \rightarrow \Lambda \bar{\Lambda}$

The first observation of transverse polarization of $\Lambda$ in $J / \psi \rightarrow \Lambda \bar{\Lambda}$ was reported by BESIII in 2018 [18]. For the $p \pi^{-} \bar{p} \pi^{+}$final state, 420,593 events were observed with an estimated background content of $399 \pm 20$ events. The relative phase $\Delta \Phi$ was determined to be $(42.4 \pm 0.6 \pm 0.5)^{\circ}$, corresponding to a maximum transverse polarization $P_{y}$ of about $25 \%$, shown in Fig. 1(b). The moment $\mu$ related to the polarization, shown in Fig. 1(a), is calculated in 50 bins of $\cos \theta_{\Lambda}$ as a cross-check of the fit results. The non-zero polarization enabled the first independent determination of decay parameters of $\Lambda$ and $\bar{\Lambda}$, and a measurement of the CP-odd observable $A_{C P}^{\Lambda}=-0.006 \pm$ $0.012 \pm 0.007$.

To give a more precise result for the decay parameter, an average is calculated $\langle\alpha>=$ $\frac{\alpha-\bar{\alpha}}{2}=0.754 \pm 0.003 \pm 0.002$. This value deviates by $17 \%$ from the long-standing, previous PDG average [19] that has been used in many measurements of $\Lambda / \bar{\Lambda}$ polarization. The CLAS experiment has since re-analyzed its data on $\gamma p \rightarrow \Lambda K$ finding $\alpha_{\Lambda}=0.721(6)(5)$ [20]. While also larger than the previous PDG value, it is still inconsistent with the BESIII result. Further, independent measurements are therefore needed to resolve the discrepancy.
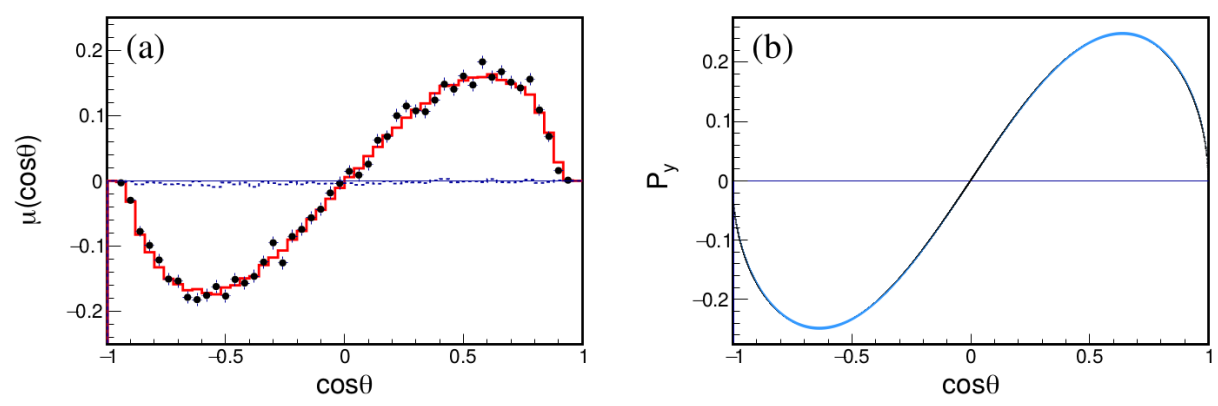

Figure 1: (a) Moment $\mu$ calculated using data uncorrected for efficiency. The black points with error bars represent data, the red histogram represents the fit results, and the blue line represents an unpolarized distribution. (b) Angular dependence of the polarization $P_{y}$ of $\Lambda$ as determined by the MLL fit.

\section{$5.2 e^{+} e^{-} \rightarrow J / \psi, \psi^{\prime} \rightarrow \Sigma^{+} \bar{\Sigma}^{-}$}

BESIII has performed a DT measurement of both $J / \psi \rightarrow \Sigma^{+} \bar{\Sigma}^{-}$and $\psi^{\prime} \rightarrow \Sigma^{+} \bar{\Sigma}^{-}$[21], observing spin polarization of $\Sigma^{+}$for the first time in both cases. The value of $\alpha_{J / \psi}$ is found to be positive while $\alpha_{\psi}$ is found to be negative. The non-zero value of $\Delta \Phi$ means that the decay parameters of both $\Sigma$ and $\bar{\Sigma}$ could be determined, leading to the first CP-test in $\Sigma^{+}$decays $A_{C P}^{\Sigma^{+}}=-0.004 \pm 0.037 \pm 0.010$. The SM prediction for this value is $A_{C P}^{\Sigma^{+}} \sim 3.6 \times 10^{-6}[14]$.

$5.3 e^{+} e^{-} \rightarrow J / \psi \rightarrow \Xi \bar{\Xi}$

Processes with sequential decays such as $J / \psi \rightarrow \Xi^{-} \bar{\Xi}^{+} \rightarrow \Lambda \pi^{-} \bar{\Lambda} \pi^{+} \rightarrow p \pi^{-} \pi^{-} \bar{p} \pi^{+} \pi^{+}$are important as they allow for measurements of both decay parameters and direct extraction of the weak phase difference. In a recent paper, BESIII has performed a DT analysis of this reaction where the full event topology is reconstructed [22]. A total of 73,244 signal events are found, 
and the background content is estimated to be $187 \pm 16$ events. To fully describe the production and two-step decay, a total of nine helicity angles are needed, and there are eight parameters to be determined $\omega_{\Xi}:=\left(\alpha_{\psi}, \Delta \Phi, \alpha_{\Xi}, \phi_{\Xi}, \bar{\alpha}_{\Xi}, \bar{\phi}_{\Xi}, \alpha_{\Lambda}, \bar{\alpha}_{\Lambda}\right)$. $\Xi^{-}$is observed to be polarized and the decay parameters for both $\Xi^{-}$and $\bar{\Xi}^{+}$are determined for the first time. The decay parameters for $\Lambda$ and $\bar{\Lambda}$ are also determined, and their average $\left\langle\alpha_{\Lambda}>=0.760 \pm 0.006 \pm 0.003\right.$ is found to be consistent with the BESIII result found in $J / \psi \rightarrow \Lambda \bar{\Lambda}$. Three independent CP-tests are performed: $\left.\left.A_{C P}^{\Xi}=(6.0 \pm 13.4 \pm 5.6) \times 10^{-3}\right), A_{C P}^{\Lambda}=(-3.7 \pm 11.7 \pm 9.0) \times 10^{-3}\right)$, and $\left(\xi_{P}-\xi_{S}\right) \Xi^{-} \rightarrow \Lambda \pi^{-}=$ $(1.2 \pm 3.4 \pm 0.8) \times 10^{-2} \mathrm{rad}$. Of particular note is that the weak phase difference is extracted for the first time for any baryon. The SM expectation for this value is $(-1.9 \pm 1.6) \times 10^{-4} \mathrm{rad}$ [13]

\section{$5.4 e^{+} e^{-} \rightarrow \psi^{\prime} \rightarrow \Omega \bar{\Omega}$}

According to the quark model, the $\Omega$ has spin $J=3 / 2$. This has been tested experimentally, but only under the assumptions that the $\Xi_{c}$ spin $J=1 / 2$ [23] and never in a model independent way.

BESIII has analysed the process $\psi^{\prime} \rightarrow \Omega^{-} \bar{\Omega}^{+}$through ST measurements of the decay sequence $\Omega^{-} \rightarrow \Lambda K^{-}, \Lambda \rightarrow p \pi^{-}$and its charge conjugate [24]. Both the spin- $1 / 2$ and $-3 / 2$ formalisms are fitted to data, and the spin-3/2 hypothesis is found to be clearly favored. Thus the quark model spin is confirmed model-independently for the first time. Spin-3/2 particles can have both vector $\left(r_{1}\right)$, quadrupole $\left(r_{6}, r_{7}, r_{8}\right)$, and octupole $\left(r_{10}, r_{11}\right)$ polarizations [1]. The values of these operators are determined through a MLL fit, and their angular dependence is shown in Fig. 2.

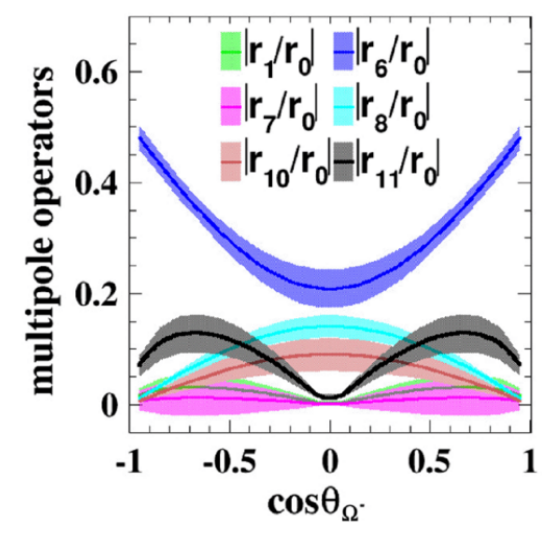

Figure 2: Angular dependence of the $\Omega^{-}$polarizations $r_{i}$ in $\psi^{\prime} \rightarrow \Omega \bar{\Omega}$. The solid lines represent the central value and the shade areas represent $1 \sigma$ deviations.

\section{Outlook}

With the $10^{10} \mathrm{~J} / \psi$ events already collected at BESIII, the statistical precision on the CP-tests could potentially be reduced by a factor of about 2.7. Future Super Charm-Tau factories currently in planning $[25,26]$ may produce more than $10^{12} \mathrm{~J} / \psi$ events, and if a polarized electron beam could be used, the statistical precision at such facilities could be pushed down to the level of the SM predictions [27]. 


\section{References}

[1] A.Z. Dubnickova, S. Dubnicka and M.P. Rekalo, Investigation of the nucleon electromagnetic structure by polarization effects in $e+e-\longrightarrow N$ anti- $N$ processes, Nuovo Cim. A 109 (1996) 241.

[2] G. Gakh and E. Tomasi-Gustafsson, General analysis of polarization phenomena in $e++e \rightarrow n+n^{-}$for axial parametrization of two-photon exchange, Nuclear Physics A 771 (2006) 169.

[3] H. Czyż, A. Grzelińska and J.H. Kühn, Spin asymmetries and correlations in $\Lambda$-pair production through the radiative return method, Phys. Rev. D 75 (2007) 074026.

[4] G. Fäldt, Entanglement in joint $\Lambda \bar{\Lambda}$ decay, Eur. Phys. J. A 51 (2015) 74 [1306.0525].

[5] G. Fäldt, Polarization observables in the $e^{+} e^{-} \rightarrow \bar{\Lambda} \Lambda$ reaction, Eur. Phys. J. A 52 (2016) 141 [1602.02532].

[6] G. Fäldt and A. Kupsc, Hadronic structure functions in the $e^{+} e^{-} \rightarrow \bar{\Lambda} \Lambda$ reaction, Phys. Lett. B 772 (2017) 16 [1702.07288].

[7] KTEV collaboration, Measurements of direct CP violation, CPT symmetry, and other parameters in the neutral kaon system, Phys. Rev. D 67 (2003) 012005.

[8] J. Batley, R. Dosanjh, T. Gershon, G. Kalmus, C. Lazzeroni, D. Munday et al., A precision measurement of direct cp violation in the decay of neutral kaons into two pions, Physics Letters B 544 (2002) 97.

[9] E. Abouzaid, M. Arenton, A.R. Barker, M. Barrio, L. Bellantoni, E. Blucher et al., Precise measurements of direct cp violation, cpt symmetry, and other parameters in the neutral kaon system, Phys. Rev. D 83 (2011) 092001.

[10] H. Gisbert and A. Pich, Direct CP violation in $\$\left\{k^{\wedge} O \backslash\right.$ to $\backslash$ pi $\left.\backslash p i\right\} \$$ : Standard model status, .

[11] J.F. Donoghue, X.-G. He and S. Pakvasa, Hyperon decays and cp nonconservation, Phys. Rev. D 34 (1986) 833.

[12] T.D. Lee and C.N. Yang, General partial wave analysis of the decay of a hyperon of spin 1/2, Phys. Rev. 108 (1957) 1645.

[13] J. Tandean and G. Valencia, CP violation in hyperon nonleptonic decays within the standard model, Phys. Rev. D 67 (2003) 056001 [hep-ph/0211165].

[14] J. Tandean, New physics and CP violation in hyperon nonleptonic decays, Phys. Rev. D 69 (2004) 076008 [hep-ph/0311036].

[15] HYPERCP collaboration, Search for CP violation in charged-Xi and Lambda hyperon decays, Phys. Rev. Lett. 93 (2004) 262001 [hep-ex/0412038]. 
[16] P.D. Barnes, G. Diebold, G. Franklin, B. Quinn, R. Schumacher, J. Seydoux et al., Observables in high-statistics measurements of the reaction $p_{\bar{p}} \rightarrow \Lambda^{-} \Lambda$, Phys. Rev. C 54 (1996) 1877.

[17] E. Perotti, G. Fäldt, A. Kupsc, S. Leupold and J.J. Song, Polarization observables in $e^{+} e^{-}$ annihilation to a baryon-antibaryon pair, Phys. Rev. D 99 (2019) 056008.

[18] BESIII collaboration, Polarization and Entanglement in Baryon-Antibaryon Pair Production in Electron-Positron Annihilation, Nature Phys. 15 (2019) 631 [1808. 08917].

[19] Particle Data Group collaboration, Review of Particle Physics, Phys. Rev. D 98 (2018) 030001.

[20] D.G. Ireland, M. Döring, D.I. Glazier, J. Haidenbauer, M. Mai, R. Murray-Smith et al., Kaon photoproduction and the $\Lambda$ decay parameter $\alpha_{-}$, Phys. Rev. Lett. 123 (2019) 182301.

[21] BESIII collaboration, $\Sigma^{+}$and $\bar{\Sigma}^{-}$polarization in the $J / \psi$ and $\psi(3686)$ decays, Phys. Rev. Lett. 125 (2020) 052004.

[22] BESIII collaboration, Weak phases and CP-symmetry tests in sequential decays of entangled double-strange baryons, 2105.11155.

[23] BABAR collaboration, Measurement of the spin of the Omega- hyperon at BABAR, Phys. Rev. Lett. 97 (2006) 112001 [hep-ex/0606039].

[24] BESIII collaboration, Model-independent determination of the spin of the $\Omega^{-}$and its polarization alignment in $\psi(3686) \rightarrow \Omega^{-} \bar{\Omega}^{+}$, Phys. Rev. Lett. 126 (2021) 092002.

[25] E.B. Levichev, A.N. Skrinsky, G.M. Tumaikin and Y.M. Shatunov, Electron-positron beam collision studies at the budker institute of nuclear physics, Physics-Uspekhi 61 (2018) 405.

[26] Q. Luo and D. Xu, Progress on Preliminary Conceptual Study of HIEPA, a Super Tau-Charm Factory in China, in Proc. 9th International Particle Accelerator Conference (IPAC'18), Vancouver, BC, Canada, April 29-May 4, 2018, no. 9 in International Particle Accelerator Conference, (Geneva, Switzerland), pp. 422-424, JACoW Publishing, June, 2018, DOI.

[27] A. Kupsc, Precision hyperon physics at J/ $\psi$ and $\psi^{\prime}$ factories, PoS CHARM2020 (2021) 009. 